\title{
Is the Presence of Microalbuminuria a Relevant Marker of Kidney Disease?
}

\author{
Richard J. Glassock
}

Published online: 5 August 2010

(C) The Author(s) 2010. This article is published with open access at Springerlink.com

\begin{abstract}
Levels of urinary albumin excretion that are below the usual limit of detection by qualitative testing, but are above normal levels (microalbuminuria; MA), can be readily identified by simple measures, such as the urinary albumin to creatinine ratio in untimed urine samples. Such measurements, particularly when combined with assessment of estimated glomerular filtration rate (eGFR), have utility as biomarkers for enhanced risk of all-cause mortality, cardiovascular events, progressive chronic kidney disease, and endstage renal disease in diabetic and nondiabetic subjects. However, it is controversial whether "isolated" MA (MA in the absence of a clear reduction in eGFR, urine sediment abnormalities, or structural renal disease) should be regarded as kidney disease. Such MA could also be regarded as a manifestation of a diffuse endothelial (microvascular) injury and thereby collateral kidney damage. This article reviews the current evidence concerning MA as a marker of kidney disease or kidney damage.
\end{abstract}

Keywords Microalbuminuria - Chronic kidney disease . Cardiovascular events · End-stage kidney disease . Albuminuria - Estimated glomerular filtration rate . All-cause mortality

\footnotetext{
R. J. Glassock

David Geffen School of Medicine at UCLA,

Los Angeles, CA, USA

R. J. Glassock $(\bowtie)$

8 Bethany,

Laguna Niguel, CA 92677, USA

e-mail: glassock@cox.net

\section{Introduction}

Microalbuminuria (MA) was delineated many years ago to describe the appearance of small quantities of albumin, too little to detect by standard "dipstick" methods, in urine samples from subjects with diabetes $[1,2]$. According to recent guidelines, MA may be defined as the urinary excretion of $30-300 \mathrm{mg} / \mathrm{d}$ in a timed urine collection in adults [3]. When spot urine samples are used, the recommended definitions of MA are the excretion of $17-250 \mathrm{mg} / \mathrm{g}$ of creatinine in men and $25-355 \mathrm{mg} / \mathrm{g}$ of creatinine in women. This measurement is known as the urinary albumin to creatinine ratio (UACR). The gender-associated differences in definition result from variation in daily creatinine generation-females have lower creatinine generation than males [4]. In addition, variations in the quantification of albuminuria for these definitions of MA may need to be applied to the elderly (who have greatly decreased creatinine generation) or to excessively muscular individuals (who have high creatinine generation) [3]. Urinary albumin concentration in untimed urinary collections may also be used to define MA - values of 3-30 mg/dL generally are regarded as fulfilling the definition. Albumin in urine is most often measured by immuno-turbidimetry using antibodies reactive with intact albumin. Alternatively, high-pressure liquid chromatography can be used, but this method also measures nonimmunologically reactive albumin and albumin fragments, which yields higher values than immuno-turbidimetry [5]. In general, MA corresponds to a "trace" reading on dipstick testing of urine, but results depend on the degree of urine concentration. Dye-impregnated strips that have enhanced sensitivity for albumin are also available [6]. Simple, portable, "point-of-service" instruments that measure small 
amounts of albumin make assessment of MA readily available, reliable, and inexpensive [7].

The precise organ of urinary albumin remains somewhat controversial. The dominant viewpoint is that albumin is normally filtered by the glomeruli in small but significant amounts, about 1 to $2 \mathrm{mg} / \mathrm{min}(\sim 2 \mathrm{~g} / \mathrm{d})$, but the great majority $(99 \%)$ of filtered albumin is reabsorbed and degraded by the proximal tubule, leaving less than about $5 \mu \mathrm{g} / \mathrm{min}(7 \mathrm{mg} / \mathrm{d})$ to be excreted [8]. A minority view is that larger amounts of albumin are filtered, as much as $200 \mathrm{~g} / \mathrm{d}$, but a retrieval pathway in the proximal tubule reclaims the filtered albumin intact and delivers it to the circulation, except for small amounts of intact and partially or fully degraded albumin [9]. In pathologic states, the glomeruli may become increasingly permeable to circulating albumin by virtue of disturbances in endothelial cell function, basement membrane abnormalities, or podocyte (visceral epithelial cell) disorders. Currently, the focus is on disturbances of endothelial or podocyte function as causes of excessive albumin glomerular permeability, rather than disturbances of basement membrane physiology [8]. Albumin is negatively charged at physiologic $\mathrm{pH}$ and may be impeded in transglomerular passage by the anionic residues in endothelial cell, glomerular basement membrane, or podocyte [8]. However, the existence of a charge-selective permeability barrier has been challenged [10]. A reduction in proximal tubule reclamation or reabsorption of filtered albumin can also cause albuminuria [9]. Finally, physicochemical alterations in the circulating albumin molecule can affect its permeation through the glomerular capillary wall, by altering the shape or possibly the electrical charge [11]. Thus, the mechanisms underlying increased urinary albumin excretion are complex and it is often difficult to ascribe MA in specific patients to a unique pathophysiologic process; however, endothelial cell dysfunction, a podocytopathy, or both seem to underlie most instances. Nevertheless, it remains possible that alterations in proximal tubular function may contribute to albuminuria in some circumstances, such as during poorly controlled glycemia in diabetes mellitus. These considerations are important when one attempts to relate albumin excretion rates to the definition of kidney disease, because albuminuria arising from diffuse endothelial cell injury (eg, in atherosclerosis and hypertension) might best be regarded as a sign of a systemic disease process, affecting many organs and systems, rather than one originating in the kidneys. Alternatively, albuminuria arising from a podocyte dysfunction could legitimately be regarded as a manifestation of a kidney disease. Regardless of the underlying mechanisms and interpretation of the pathophysiologic meaning of albuminuria, important associations exist between the quantities of albumin excreted in the urine and progressive chronic kidney disease (CKD) and cardiovascular (CV) events.

\section{Microalbuminuria: Association with Progressive "Generic" CKD and CV Events}

Microalbuminuria, as defined above, has been consistently associated with an increased likelihood of progression of generic CKD to more advanced stages or even to end-stage renal disease (ESRD) in large epidemiologic studies [12 •, $13 \bullet, 14 \cdot \bullet, 15]$. These observations have been used to justify the inclusion of MA in the definition of generic CKD in the original classification schema of the Kidney Disease Outcome Quality Initiative (KDOQI) of the National Kidney Foundation (NKF) in 2002 [3]. Indeed, the presence of MA (defined as UACR of $30-299 \mathrm{mg} / \mathrm{g}$ creatinine without gender or age adjustments) as the sole reason for diagnosing generic CKD accounted for $90 \%$ of those with stage 1 CKD and $87 \%$ of those with stage 2 CKD in the US National Health and Nutrition Examination Survey (NHANES) conducted from 1999 to 2004 [16]. In this cohort $(N=13,233), 6.8 \%$ had self-reported diabetes and $27 \%$ had diagnosed hypertension. Thus, the great majority of subjects designated as having stage 1 or $2 \mathrm{CKD}$ in NHANES had isolated MA (MA without a clear reduction in estimated glomerular filtration rate [eGFR], urine sediment changes, or structural kidney disease) as the defining abnormality. It is natural to ask whether such isolated MA is sufficient reason to designate a person as having generic CKD.

As shown by a recent, very large, collaborative metaanalysis (Chronic Kidney Disease Prognosis Consortium; $N=$ 105,872 subjects), the hazard ratio (HR) for all-cause mortality and cardiovascular mortality (adjusted for the effects of age, ancestry, history of CV disease, systolic blood pressure, diabetes, smoking, and total cholesterol concentration) rises progressively above a UACR of about $10 \mathrm{mg} / \mathrm{g}$ $[17 \cdot \bullet]$. In subjects with normal renal function $(\mathrm{eGFR}=90$ $104 \mathrm{~mL} / \mathrm{min} / 1.73 \mathrm{~m}^{2}$ ), the HR for CV mortality is 1.63 at a UACR of $10-29 \mathrm{mg} / \mathrm{g}, 1.82$ at $30-299 \mathrm{mg} / \mathrm{g}$, and 4.77 at greater than $300 \mathrm{mg} / \mathrm{g}$. A decrease in eGFR magnifies the effect of an increased UACR on both all-cause and CV mortality, especially at an eGFR of $45 \mathrm{~mL} / \mathrm{min} / 1.73 \mathrm{~m}^{2}$ or lower. Thus, there is little or no doubt that increased albumin exertion, even below the conventional MA range, is associated with an increased likelihood for mortality, both all-cause and CV-related. The causal direction for this association cannot be determined from this epidemiologic data. Is elevated albumin excretion a biologic marker of underlying systemic disease (eg, cancer, atherosclerosis), or is it somehow involved in the causal pathway for fatal events, including CV disease?

Similarly, the risk of developing a progressive form of CKD is linked somehow to the presence of elevated albumin exertion. A large, community-based study from Alberta, Canada, $(N=920,875)$ showed that subjects with 
normal or nearly normal renal function (eGFR $\geq 60 \mathrm{~mL} / \mathrm{min} /$ $1.73 \mathrm{~m}^{2}$, average age 46 years) and MA had event rates of ESRD of 1.5 and doubling of serum creatinine of 2.8 times those of subjects with "normal" albumin excretion $(<30 \mathrm{mg} / \mathrm{g}$ UACR $)$ [14••]. Absolute events rates were low: 0.06 per 1000 person-years of follow-up for ESRD in the normo-albuminuric group versus 0.09 per 1000 patientyears for the MA group. A lowered eGFR magnified the effect of MA on the occurrence of ESRD and doubling of the serum creatinine, especially at levels below $45 \mathrm{~mL} / \mathrm{min} /$ $1.73 \mathrm{~m}^{2}$. In another community-based study $(N=65,589$ adults; $3.3 \%$ diabetic; average age, 50.1 years), researchers noted that the likelihood of developing (and surviving long enough) to receive treatment for ESRD among subjects with MA and eGFR $\geq 60 \mathrm{~mL} / \mathrm{min} / 1.73 \mathrm{~m}^{2}$ was 27 times that of subjects with normo-albuminuria and similar eGFR levels [13•]. A reduced eGFR greatly magnified the effect of MA on risk for ESRD. Subjects with an eGFR less than $60 \mathrm{~mL} / \mathrm{min} / 1.73 \mathrm{~m}^{2}$ and MA had a 5.4-fold to 81 -fold increased risk of ESRD compared to those with an eGFR $\geq$ $60 \mathrm{~mL} / \mathrm{min} / 1.73 \mathrm{~m}^{2}$, depending on the degree of decrease in eGFR. Thus, the addition of MA to eGFR greatly enhances the ability to detect and quantify risk of progressive CKD, particularly when eGFR is less than $60 \mathrm{~mL} / \mathrm{min} / 1.73 \mathrm{~m}^{2}$. These observations call for a revision of the 2002 version of the KDOQI-NKF classification schema for CKD that does not include albuminuria for diagnosis of stage 3 or above CKD [3]

Taken together, these findings from epidemiologic studies using large databases strongly support the view that MA needs to be considered as a "biomarker" of adverse outcomes, even among those subjects with normal or nearly normal renal function. However, the strength of this association between MA and outcomes may vary by subject age and underlying disease (eg, diabetic or nondiabetic) or by concomitant illness known to influence albumin excretion rates (eg, obesity). For example, in the aforementioned meta-analysis conducted by the Chronic Kidney Disease Prognosis Consortium [17••], the pooled estimates of fully adjusted HR for CV mortality tended to be higher in those younger than 65 years of age compared to those older than 65 years with apparently equivalent degrees of MA. An analysis of the impact of MA on outcomes in the oldold ( $>75$ years of age) has not been conducted; however, as stated above, UACR has a tendency to increase with normal aging, predominantly because of the effects of sarcopenia on creatinine generation, not necessarily because of an absolute increase in albumin excretion with aging. Furthermore, the association of MA with adverse outcomes in subjects with normal or nearly normal renal function (eGFR $\geq 60 \mathrm{~mL} / \mathrm{min} / 1.73 \mathrm{~m}^{2}$ ), after adjustment for the effects of aging may simply reflect the overall burden of atherosclerotic vascular disease in this group of subjects. As recently pointed out by Kalaitzidis and Bakris [18], untangling the influences of metabolic disturbances and hypertension in diabetic and nondiabetic subjects with concomitant MA can be problematical. It is still uncertain if $\mathrm{MA}$ is an integral part of the pathophysiologic pathways for $\mathrm{CV}$ disease or simply a bystander.

\section{Microalbuminuria: Association with Progression of "Specific" CKD}

The sine qua non of "specific" forms of CKD in which MA is present is that of diabetes mellitus. Indeed, the concept of MA arose from studies of the natural history of diabetic nephropathy during the period 1981 to 1982 [1, 2]. Shortly after its initial description in type 1 diabetes, MA was believed to be a harbinger of overt diabetic nephropathy and progressive $\mathrm{CKD}$. We now recognize that MA in type 1 diabetes is a dynamic process with frequent spontaneous regression to normo-albuminuria $[19,20]$. We also recognize the wide array of extra-renal factors that can influence albumin excretion, such as obesity, age, gender, distant inflammation, and certain drugs (eg, rosuvastatin) $[21 \cdot, 22$, 23]. Furthermore, studies done at the Joslin Clinic over many years indicate that more advanced stages of CKD are not uniformly associated with progression of MA to overt microalbuminuria [19, 24]. Pathologic abnormalities indicative of diabetic nephropathy (eg, increased mesangial fractional volume) precede the development of MA, and very clearly renal functional decline can occur in the absence of MA, at least in type 1 diabetes [25]. Type 2 diabetes may be another story, because the relationship of MA and renal functional decline is not as well studied or understood as in type 1 diabetes, and this relationship is confounded by concomitant accelerated atherosclerotic macrovascular disease. Nevertheless, several studies showed that progression of type 2 diabetes can occur in the absence of MA [21, $22-26]$. Interventions designed specifically to reduce MA have not consistently shown a reduction of later progression to advanced CKD or ESRD. Some recent interventional trials (eg, the Avoiding Cardiovascular Events through Combination Therapy in Patients Living with Systolic Hypertension [ACCOMPLISH] trial) involving type 2 diabetic subjects showed that the change in albumin excretion rates can be disassociated from risk of progression to ESRD under the influence of combined renin-angiotensin system blockade and calcium channel antagonism [21, 27•]. Taken together, these observations in type 1 and type 2 diabetes suggest, but do not prove, that MA may not be a reliable marker of kidney disease or its progression. 


\section{Microalbuminuria: A Synthesis of its Relevance to Kidney Disease}

Among patients with well-established kidney disease (ie, eGFR $<45-50 \mathrm{~mL} / \mathrm{min} / 1.73 \mathrm{~m}^{2}$ ), the magnitude of albuminuria can be viewed as a marker of a more progressive course and a more rapid loss of renal function (a "risk marker" rather than a "risk factor") for both diabetic and nondiabetic renal disease [28, 29]. This finding may well be a manifestation of "lead-time bias," in that those with lower levels of albumin excretion have disease in its earlier stages and thus a greater reserve of functioning nephrons, whereas those with higher levels of albuminuria have no reserves and with each loss of nephrons, a corresponding decline in renal function becomes evident. In this sense, the level of albumin excretion is a relevant marker of kidney disease and risk of its progression. A conundrum exists when renal function is entirely or nearly normal: no overt structural renal disease is evident, yet albumin excretion is elevated into the MA range. Such patients might be described as having "isolated" MA. No doubt this circumstance somehow predisposes to, or is a marker of a predisposition to, $\mathrm{CV}$ disease. The exact pathophysiologic connection between isolated MA and $\mathrm{CV}$ disease is not fully clarified. For example, albuminuria correlates with parameters of aortic stiffness and carotid plaque formation but not with carotid intima-media thickness as a surrogate for widespread atherosclerosis [30]. On the other hand, MA does correlate with signs of altered endothelial function, such as endothelium-dependent vascular dilatation [31-33]; however, it is often difficult to disentangle the effects of alterations in renal function and concomitant metabolic and dyslipidemia states in identifying direct relationships between MA and endothelial dysfunction. Isolated MA may well be a biomarker of widespread vascular injury and atherosclerotic burden. In this sense, it is not measuring a "kidney disease" per se, but only a secondary and indirect effect of a distant disease process on kidney physiology. Although this conclusion may appear to be semantic rather than realitybased, it has immediate relevance for the diagnosis of CKD using current classification schema (ie, KDOQICKD). Stage 1 and $2 \mathrm{CKD}$ in this schema cannot be reliably distinguished on the basis of eGFR alone because current creatinine-based eGFR formulas are imprecise. Whether newer cystatin C-based formulas will correct this deficiency remains to be seen; preliminary findings are encouraging, but not uniformly so [34, 35]. Most cases of stage 1 and 2 CKD are currently defined on the basis of MA, typically in an isolated form. This definition is useful to stratify the eventual risk of $\mathrm{CV}$ events and all-cause mortality; however, does it have the same utility for defining the presence of and risk for progressive CKD and eventual ESRD at all levels of eGFR and in all ages? Current evidence suggests that the utility of MA in predicting renal outcomes is most pronounced in those with well-established kidney disease (eg, eGFR $<45-50 \mathrm{~mL} / \mathrm{min} /$ $1.73 \mathrm{~m}^{2}$ ) and in younger subjects (eg, $<65$ years of age). In my opinion, whether it is appropriate to regard isolated MA as equivalent to kidney disease is still an open question. A compromise position would be to regard this laboratory finding as an indication of kidney "damage" rather than giving it the more ominous label of "disease." This issue is not trivial-about $30 \%$ of those diagnosed as having $\mathrm{CKD}$ in epidemiologic studies have their kidney disease identified by the finding of MA and eGFR $\geq 60 \mathrm{~mL} / \mathrm{min} / 1.73 \mathrm{~m}^{2}$ in the absence of a selfreported diagnosis of diabetes.

Disclosure The author has received consulting fees from FibroGen. No other potential conflict of interest relevant to this article was reported.

Open Access This article is distributed under the terms of the Creative Commons Attribution Noncommercial License which permits any noncommercial use, distribution, and reproduction in any medium, provided the original author(s) and source are credited.

\section{References}

Papers of particular interest, published recently, have been highlighted as:

- Of importance

•• Of major importance

1. Svendsen PA, Oxenball B, Christiansen JS: Microalbuminuria in diabetic patients - a longitudinal study. Acta Endocrinol Suppl (Copenhagen) 1981, 242:53-54.

2. Viberti GC, Hill RD, Jarrett RJ, et al.: Microalbuminuria as a predictor of clinical nephropathy in insulin-dependent diabetes mellitus. Lancet 1982, 1:1430-1432.

3. National Kidney Foundation: Kidney Disease Outcomes Quality Initiative. Clinical Practice Guidelines for Chronic Kidney Disease; Evaluation, Classification and Stratification. Am J Kidney Disease 2002, 39(Suppl 1):S1-S264.

4. Epstein M: Aging and the kidney. J Am Soc Neprhol 1996, $7: 1106-1122$

5. Comper W, Osicka TM: Detection of urinary albumin. Adv Chronic Kidney Dis 2005, 12:170-176.

6. Leong SO, Lui KF, Ng WY, Thai AC: The use of semi-quantitative urine test-strip (Micral test) for microalbuminuria screening in patients with diabetes mellitus. Singapore Med J 1998, 39:101-103.

7. Sarafidis PA, Riehle J, Bogojevic Z, et al.: A comparative evaluation of various methods for microalbuminuria screening. Am J Nephrol 2009, 28:324-329.

8. Haraldsson B, Nystrom J, Deen WM: Properties of the glomerular barrier and mechanisms of proteinuria. Physiol Rev 2008, 88:451-487. 
9. Russo LM, Sandoval RM, Campos SB, et al.: Impaired tubular uptake explains albuminuria in early diabetic nephropathy. J Am Soc Nephrol 2009, 20:489-494.

10. Russo LM, Bakris GL, Comper WD: Renal handling of albumin: a critical review of basic concepts and perspective. Am J Kidney Dis 2002, 39:899-919.

11. Bundschuh I, Jackle-Meyer I, Luneberg E, et al.: Glycation of serum albumin and its role in renal protein excretion and the development of diabetic nephropathy. Eur J Chem Clin Biochem 1992, 30:651-656.

12. •- James MT, Hemmelgarn BR, Tonelli M: Early recognition and prevention of chronic kidney disease. Lancet 2010, 375:12961309. This is a seminal review article that summarizes knowledge regarding detection and prevention of chronic kidney disease in a succinct and readable fashion.

13. - Hallan SI, Ritz E, Lydersen S, et al.: Combining GFR and albuminuria to classify CKD improves prediction of ESRD. J Am Soc Nephrol 2009, 20:1069-1077. This is an excellent article that clearly shows the interactions between eGFR and albuminuria in determining risk for progressive kidney disease.

14. •- Hemmelgarn BR, Manns BJ, Lloyd A, et al.; Alberta Kidney Disease Network: Relation between kidney function, proteinuria and adverse outcomes. JAMA 2010, 301:423-429. This seminal article describes the relationship between eGFR and albuminuria or proteinuria and risk for cardiovascular events and progressive kidney disease.

15. van der Velde M, de Jong PE, Gansevoort RT: Comparison of the yield of differing screening approaches to detect chronic kidney disease. Nephrol Dial Transplant 2010 (Epub ahead of print).

16. Coresh J, Selvin E, Stevens LA, et al.: Prevalence of chronic kidney disease in the United States. JAMA 2007, 298:2018-2047.

17. •• Chronic Kidney Disease Prognosis Consortium: Association of estimated glomerular filtration rate and albuminuria with all-cause and cardiovascular mortality in general population cohorts: a collaborative meta-analysis. Lancet 2010, 375:2073-2081. This landmark study involved a very large collection of databases and meta-analyses. It characterizes the relationships of eGFR and albuminuria or proteinuria and prognosis for all-cause mortality and cardiovascular mortality.

18. Kalaitzidis RG, Bakris GL: Serum creatinine vs albuminuria as biomarkers for the estimation of cardiovascular risk. Curr Vasc Pharmacol 2010 (Epub ahead of print).

19. Perkins BA, Ficociello LH, Silva KH, et al.: Regression of microalbuminuria in type 1 diabetes. N Engl J Med 2003, 348:2285-2293.

20. Steinke JM, Sinaiko AR, Kramer MS, et al.; International Diabetic Nephropathy Study Group: The early natural history of nephropathy in type 1 diabetes: III. Predictors of 5-year urinary albumin excretion rate patterns in initially normoalbuminuric patients. Diabetes 2005, 54:2164-2171.

21. - Glassock R: Debate: CON position. Should microalbuminuria ever be considered as a renal endpoint in any clinical trial? Am J Nephrol 2010, 31:462-465. This article forms part of a "debate" regarding the significance of microalbuminuria as a surrogate end point in prospective clinical trials.
22. Bello AK, de Zeeuw D, El Nahas M, et al.: Impact of weight change on albuminuria in the general population. Nephrol Dial Transplant 2007, 22:1619-1627.

23. Vlassara H, Torreggiani M, Post JB, et al.: Role of oxidants/ inflammation in declining renal function in chronic kidney disease and normal aging. Kidney Int Suppl 2009, 114:S3-S11.

24. Perkins BA, Ficociello LH, Roshan B, et al.: In patients with type 1 diabetes and new-onset microalbuminuria the development of advanced chronic kidney disease may not require progression to proteinuria. Kidney Int 2010, 77:57-64.

25. Mauer M, Zinman B, Gardiner R, et al.: Renal and retinal effects of enalapril and losartan in type 1 diabetes. N Engl J Med 2009, $361: 40-51$

26. Ito $\mathrm{H}$, Takeuchi $\mathrm{Y}$, Ishida $\mathrm{H}$, et al.: High frequencies of diabetic micro- and macroangiopathies in patients with type 2 diabetes mellitus with decreased estimated glomerular filtration rate and normoalbuminuria. Nephrol Dial Transplant 2010, 25:1161-1167.

27. • Bakris GL, Sarafidis PA, Weir MR, et al.; ACCOMPLISH Trial Investigators: Renal outcomes with different fixed dose combination therapies in patients with hypertension at high risk for cardiovascular events (ACCOMPLISH): a prespecified secondary analysis of a randomised clinical trial. Lancet 2010, 375:11731181. This seminal clinical trial showed that a change in microalbuminuria can be dissociated from renal outcomes with fixed-dose combined therapy in high-risk patients.

28. Lorenzo V, Saracho R, Zamora J, et al.: Similar renal decline in diabetic and non-diabetic patients with comparable degrees of albuminuria. Nephrol Dial Transplant 2010, 25:835-841.

29. - de Jong P, Gansevoort RT: Albuminuria in non-primary renal disease: risk marker rather than risk factor. Nephrol Dial Transplant 2010, 25:656-658. This article provides a nice critical review of the status of microalbuminuria as a risk factor or risk marker.

30. Choi SW, Yun WJ, Kim HY et al.: Association between albuminuria, carotid atherosclerosis, arterial stiffness and peripheral artery disease in Korean type 2 diabetic patients. Kidney Blood Press Res 2010, 33:111-118.

31. Solbu MD, Jenssen TG, Eriksen BO, Toft I: Changes in insulin sensitivity, renal function and markers of endothelial dysfunction in hypertension - the impact of microalbuminuria: a 13 year follow-up study. Metabolism 2009, 58:408-415.

32. Silva AM, Schaan BD, Signori LU, et al.: Microalbuminuria is associated with impaired arterial and venous endothelium dependent vasodilation in patients with type 2 diabetes. J Endocrinol Invest 2010 (Epub ahead of print).

33. Foster MC, Keyes MJ, Larson MG, et al.: Relations of measures of endothelial function and kidney disease: the Framingham Heart Study. Am J Kidney Dis 2008, 52:859-867.

34. Rifkin DE, Katz R, Chonchoi M, et al.: Albuminuria, impaired kidney function and cardiovascular outcomes or mortality in the elderly. Nephrol Dial Transplant 2010, 25:1560-1567.

35. Bielby J, Divitini ML, Knuiman MW, et al.: Comparison of cystatin $\mathrm{C}$ and creatinine as predictors of cardiovascular events in a community-based elderly population. Clin Chem 2010, 56:799804. 